\title{
Bilateral Upper Limb Remote Ischemic Preconditioning Improves Anaerobic Power
}

\author{
Alexander S. Kraus ${ }^{1}$, Evan P. Pasha ${ }^{1}$, Daniel R. Machin ${ }^{1}$, Mohammed Alkatan ${ }^{1}$, Robert A. Kloner ${ }^{2}$ \\ and Hirofumi Tanaka ${ }^{*}, 1$ \\ ${ }^{I}$ Department of Kinesiology and Health Education, The University of Texas at Austin, Austin, TX 78712, USA \\ ${ }^{2}$ The Heart Institute, Good Samaritan Hospital, Los Angeles, CA 90017, USA
}

\begin{abstract}
Purpose: Ischemic preconditioning (IPC) has been used to protect myocardial cells against ischemiareperfusion injury and is recently utilized for improving exercise performance. It is unknown whether remote IPC (RIPC) to tissues not involved in exercise can induce similar exercise improvements and what "dose" of IPC is necessary to induce exercise performance benefits. This study determined if unilateral and bilateral upper limb RIPC improves lower body anaerobic power output.

Methods: Using two randomized, sham-controlled, crossover study designs, we studied 43 recreationally-active adults. For study 1, unilateral RIPC was used, while for study 2, the ischemic stimuli was increased to bilateral occlusion. After either the RIPC or sham control treatment, subjects completed four $30 \mathrm{~s}$ Wingate anaerobic tests on a Monark cycle ergometer with 2 min passive rest between trials.

Results: In the unilateral occlusion trial, peak power, mean power, and fatigue index were not different between the two conditions at every Wingate test. In the bilateral occlusion trial, mean power was greater in the RIPC condition during the first Wingate test compared with the sham control (both $p<0.05$ ). Additionally, peak power was elevated following the RIPC condition versus the sham control for the final Wingate test $(p<0.05)$.

Conclusion: Remote ischemic preconditioning applied bilaterally increased lower body power output over a series of Wingate anaerobic tests. Unilateral RIPC, however, had no effect on any of the performance variables, suggesting that there is a threshold for the amount of target tissues needed to elicit anaerobic performance benefits.
\end{abstract}

Keywords: Exercise performance, ischemia, reperfusion, Wingate anaerobic power.

\section{INTRODUCTION}

The extreme competitiveness and small margins that separate winners from losers have driven athletes to seek any substances, techniques, or means that provide them with a competitive edge [1]. In recent years, the utilization of socalled ergogenic aids is widely spread, not only in elite athletes, but also among regular exercisers. Indeed the biggest users of nutritional supplements, as well as anabolic steroids, are "gym rats" or recreationally-active adults [1]. Most of the frequently-used, athletic performance-enhancing ergogenic aids originate from clinical or medical uses targeted at patient populations to ameliorate symptoms and dysfunctions. One of the newest applications of a clinical utility applied for the purpose of aiding performance is ischemic preconditioning (IPC) $[2,3]$. Ischemic preconditioning in the clinical practice is performed by applying alternating bouts of ischemia and reperfusion typically to myocardial tissue and has been shown to delay cardiac cell injury following a subsequent ischemic insult

*Address correspondence to this author at the Department of Kinesiology and Health Education, The University of Texas at Austin, 2109 San Jacinto Blvd, D3700, Austin, TX 78712, USA; Tel: 512-232-4801;

Fax: 512-471-0946; E-mail: htanaka@austin.utexas.edu
[4]. The development of remote ischemic preconditioning (RIPC) has since provided a noninvasive, clinically applicable method for preconditioning of ischemic myocardium through remote occlusion of the artery [5-7].

IPC is very attractive as an ergogenic aid in several aspects as it is non-invasive, legal, and easy to apply, and is devoid of deleterious side-effects that other ergogenic aids often suffer. In one of the original studies to address this, bilateral lower extremity IPC improved maximal oxygen consumption by $3 \%$ in well-trained cyclists [2]. However, the effects of IPC on maximal oxygen consumption have not been replicated in subsequent studies [3, 8, 9]. A more promising application of IPC appears to be in exercise events that involve anaerobic power output as IPC has been demonstrated to increase resistance to hypoxic injury and ischemic tolerance [10]. In incremental maximal cycling tests, maximal oxygen consumption did not change but maximal workload and total exercise time increased with the IPC application [9], suggesting that IPC might have enhanced anaerobic capacity. Bilateral upper extremity IPC elicited an improvement in $100 \mathrm{~m}$ swim time in Olympic level swimmers [3]. To date, previous studies involving IPC and exercise performance have all used localized IPC, applying IPC to the tissues subsequently used for exercise performance. Few studies have examined whether a remote 
bout of IPC to tissue not involved in exercise (i.e., upper extremity IPC prior to lower body exercise) is capable of improving exercise performance [11]. Moreover, it is unknown what "dose" of RIPC is necessary to induce exercise performance benefits.

Accordingly, the primary aim of the present study was to test the hypothesis that RIPC of the upper extremity would confer systemic benefits and produce improvements in lower body anaerobic power output. Because many sporting events (e.g., basketball, soccer, ice hockey) are performed in an intermittent fashion, we implemented 4 repeated anaerobic tasks in a row, to see if the effects of RIPC could persist through multiple episodes of exercise.

\section{METHODS}

\section{Study Design}

In order to evaluate the effects of RIPC on anaerobic exercise performance, we conducted 2 different but complimentary studies. Within each study, we used a randomized, sham-controlled, single-blinded, crossover experimental design with two experimental conditions. For study 1, unilateral remote ischemic preconditioning was used, and a sham control condition involved the inflation of blood pressure cuffs to $10 \mathrm{mmHg}$. For study 2, the ischemic stimuli were increased to bilateral occlusion. Additionally, to eliminate the possibility of the low blood pressure cuff inflation inducing any ischemic conditioning effects in the sham control condition [9], the cuff was placed but was not inflated during the sham control for study 2. Other experimental procedures were identical between the 2 studies.

\section{Subjects}

A total of 43 young, healthy, recreationally-active subjects were studied in the present study. For study 1,14 adults (6 males, 8 females) participated. For study 2, a different set of 29 adults (21 males, 8 females) volunteered to participate. Selected subject characteristics are displayed in Table 1.

Exclusion criteria employed for both studies were: medication usage, regular smoking, and chronic disease as assessed by a Health Research Questionnaire. The Institutional Review Board at the University of Texas at Austin reviewed and approved the study. All volunteers gave their written informed consent before participation.

\section{Experimental Protocol}

To ensure that subjects could reliably complete the exercise protocol, all subjects performed two familiarization trials [12]. Subjects completed two $30 \mathrm{~s}$ Wingate tests separated by two minutes of passive recovery in the first familiarization, and four $30 \mathrm{~s}$ Wingate tests separated by two minutes of passive recovery in the second familiarization. The main experimental protocol was performed at least a week after the familiarization sessions. Owing to the successive familiarization sessions, there were no "order effects" observed in the main protocols.
Table 1. Selected subject characteristics.

\begin{tabular}{|l|c|c|}
\hline Characteristics & $\begin{array}{c}\text { Unilateral } \\
\text { RIPC }\end{array}$ & $\begin{array}{c}\text { Bilateral } \\
\text { RIPC }\end{array}$ \\
\hline \hline n (males, females) & $14(6,8)$ & $29(21,8)$ \\
\hline Age (years) & $22.2 \pm 5.3$ & $23.2 \pm 3.8$ \\
\hline Height (cm) & $168 \pm 7$ & $175 \pm 6$ \\
\hline Body Mass (kg) & $66.3 \pm 10.7$ & $72.2 \pm 10.6$ \\
\hline BMI (kg/m $\left.{ }^{2}\right)$ & $23.4 \pm 2.5$ & $23.5 \pm 3.5$ \\
\hline Systolic Blood Pressure (mm Hg) & $118 \pm 11$ & $119 \pm 11$ \\
\hline Diastolic Blood Pressure (mm Hg) & $67 \pm 8$ & $71 \pm 6$ \\
\hline Physical Activity (Min/Week) & & \\
\hline Low Activity & $79 \pm 75$ & $87 \pm 108$ \\
\hline Moderate Activity & $146+110$ & $106 \pm 85$ \\
\hline Vigorous Activity & $189 \pm 93$ & $219 \pm 163$ \\
\hline Total Activity & $414 \pm 192$ & $413 \pm 201$ \\
\hline
\end{tabular}

Values are expressed as means \pm SD

$\mathrm{RIPC}=$ remote ischemic preconditioning; $\mathrm{BMI}=$ body mass index

Physical activity was determined via 7-day physical activity recall.

All study activities were performed under ambient temperature $\left(22.4^{\circ} \mathrm{C}\right)$, humidity $(37 \%)$, and barometric pressure $(757 \mathrm{mmHg})$ in a controlled laboratory setting. For each testing visit, subjects reported to The University of Texas Cardiovascular Aging Research Laboratory at the same time of day each time to eliminate any potential diurnal effects on power output [13]. Additionally, subjects were fasted for at least four hours and had abstained from alcohol, caffeine, and vigorous physical activity for at least $24 \mathrm{~h}$ before testing. Upon arrival, participants sat quietly for 5 min in a controlled environment, and baseline resting blood pressure was measured (HEM-907, Omron Healthcare, Lake Forest, IL). The test protocol employed in the present study is displayed in Fig. (1).

While in the supine position, an automated inflatable cuff (E20 Rapid Cuff Inflator, D.E. Hokanson, Bellevue, WA) was positioned unilaterally on the left upper arm in study 1 . In study 2, the same blood pressure cuffs were placed bilaterally on the left and right upper arms. Participants then received four, 5 min episodes of RIPC or sham treatment, followed each by $5 \mathrm{~min}$ of reperfusion. The bilateral occlusion was performed simultaneously on the right and left arms. On the subsequent visit at the same time of the day, participants completed the identical procedure except undergoing the alternative condition (RIPC versus sham). Although participants were likely aware of pressure differences between conditions, they remained naïve to the rationale of the experiment. To ensure that the occlusion and reperfusion of tissue were properly achieved, finger temperature was monitored throughout both conditions (DTM Raw Data Acquisition, Endothelix, Houston, TX) [11]. Female subjects were studied during the same phase of the menstrual cycle [14].

Prior to the exercise protocol, subjects engaged in a 5 min warm-up. During this warm-up period, subjects began 


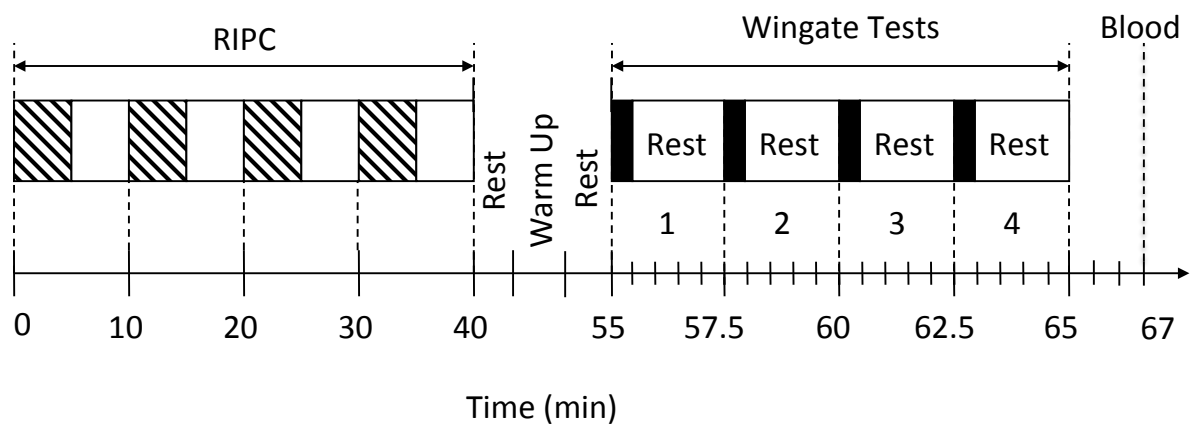

Fig. (1). Experimental test protocol involving remote ischemic pre-conditioning (RIPC). RIPC = RIPC treatment in which striped boxes represent $5 \mathrm{~min}$ of occlusion and white boxes represent $5 \mathrm{~min}$ of reperfusion. Wingate tests $=$ Wingate anaerobic performance tests in which black boxes represent $30 \mathrm{sec}$ of Wingate exercise and rest boxes represent the subsequent 2 min rest.

pedalling comfortably against no resistance at $60-75 \mathrm{rpm}$ $(\sim 50 \mathrm{~W})$ on a cycle ergometer (Monark Erogomedic $894 \mathrm{E}$ Peak Bike, Vansbro, Sweden) and executed an intermittent sprint achieving maximal voluntary rpm at minutes 2,3 , and 4 , respectively. Following the warm up, participants rested quietly for $5 \mathrm{~min}$ before performing the Wingate exercise [12].

Fifteen minutes after each experimental condition, participants completed four Wingate anaerobic power tests on a specialized cycle ergometer (Monark Erogomedic 894E Peak Bike, Vansbro, Sweden). Each test lasted $30 \mathrm{~s}$ in duration, followed by 2 min of passive rest. Subjects cycled from rest to $150 \mathrm{rpm}$, after which a frictional resistance of $9 \%$ body weight was instantly applied to the flywheel. Subjects received no verbal encouragement during each test in an attempt to minimize the investigator bias. Five minutes following the final exercise bout, blood lactate was collected (LactatePro, Arkray, Kyoto, Japan). Peak power (W), mean power $(\mathrm{W})$, and fatigue index $(\%)([$ (peak power - ending power)/peak power]*100) were calculated for each Wingate test [12]. Total (composite) power output (W)(mean power per Wingate $* 30 \mathrm{~s} * 4$ sets) and overall fatigue index $(\%)$ ([(peak power $1^{\text {st }}$ Wingate - ending power $4^{\text {th }}$ Wingate) / peak power $1^{\text {st }}$ Wingate $\left.]^{*} 100\right)$ were also calculated.

\section{Statistical Analyses}

The Kolmogorov-Smirnov test indicated a normal distribution of data. Two-way (time $\mathrm{x}$ condition) ANOVA with repeated measures and least signficant difference (LSD) post hoc analysis were used to assess differences in exercise performance variables between conditions. Univariate correlational and regressional analyses were performed to determine the associations. All data were analyzed using SPSS statistical analysis software version 22.0 (SPSS Statistics, IBM, Armonk, NY). For all analyses, significance was set apriori at $p<0.05$. Data are presented as means $\pm \mathrm{SD}$ unless indicated otherwise.

\section{RESULTS}

All subjects completed all 4 Wingate anaerobic performance tests on both trials. In both studies, peak and mean power outputs demonstrated substantial reductions from Wingate 1 to Wingate 4 tests where the fatigue index remained fairly constant. In study 1 , peak power, mean power, and fatigue index were not different between the two conditions at every Wingate test. Similarly, total (composite) power output, overall fatigue index, and blood lactate concentration were not significantly different between the two conditions (Table 2).

In study 2, which utilized bilateral RIPC, peak power was significantly greater in the RIPC condition than in the sham control for the fourth Wingate test $(p<0.05)$ (Fig. 2).

Additionally, compared with the sham control, mean power was significantly higher in the RIPC condition during the first and fourth Wingate test $(p<0.05)$ (Fig. 3).

To determine if responses to RIPC can be predicted by baseline levels of Wingate power outputs, we performed correlational analyses between the two. No significant association was found between baseline anaerobic power and the changes in power output induced by RIPC. There were no significant differences between the conditions for total (composite) power output, overall fatigue index, or blood lactate concentrations (Table $\mathbf{3}$ ).

\section{DISCUSSION}

The major finding of the present study is that bilateral remote ischemic preconditioning of the arms significantly increased lower body anaerobic exercise performance. The improvements were observed in both mean and peak power output, as assessed by the well-established Wingate anaerobic power tests. Additionally, the effects of RIPC persisted in repeated bouts of anaerobic performance. To our knowledge, this is the first study to demonstrate persistent benefits of remote ischemic preconditioning stimuli on repeated bouts of anaerobic exercise performance. In contrast to the bilateral occlusions, unilateral RIPC was not associated with any improvements in the performance variables, suggesting that there may be a threshold for the amount of remote ischemic stimuli needed to elicit anaerobic performance benefits.

In a clinical setting, RIPC has been well documented to confer a variety of positive systemic effects [10]. For example, IPC applied to the legs can prevent a decrease in brachial artery endothelial function, indicating a systemic, rather than localized, effect of RIPC [15]. To this date, studies investigating the role of IPC in exercise performance have applied IPC locally to the same musculature that was subsequently used in exercise (e.g., applying IPC to the 
Table 2. Anaerobic power outputs as measured by Wingate anaerobic power tests.

\begin{tabular}{|c|c|c|c|c|}
\hline Unilateral RIPC & Wingate 1 & Wingate 2 & Wingate 3 & Wingate 4 \\
\hline \multicolumn{5}{|l|}{ Peak Power (W) } \\
\hline Sham Control & $591.7 \pm 176.2$ & $502.1 \pm 144.2$ & $473.9 \pm 117.4$ & $451.1 \pm 121.4$ \\
\hline RIPC & $565.7 \pm 174.3$ & $513.2 \pm 182.0$ & $470.7 \pm 133.6$ & $448.0 \pm 122.6$ \\
\hline \multicolumn{5}{|l|}{ Mean Power (W) } \\
\hline Sham Control & $427.2 \pm 96.2$ & $366.0 \pm 90.0$ & $334.9 \pm 72.6$ & $318.9 \pm 73.5$ \\
\hline RIPC & $409.5 \pm 98.3$ & $363.8 \pm 92.6$ & $330.0 \pm 75.2$ & $317.9 \pm 68.2$ \\
\hline \multicolumn{5}{|l|}{ Fatigue Index (\%) } \\
\hline Sham Control & $50 \pm 10$ & $46 \pm 11$ & $49 \pm 11$ & $50 \pm 12$ \\
\hline RIPC & $48 \pm 11$ & $47 \pm 12$ & $50 \pm 12$ & $50 \pm 10$ \\
\hline Bilateral RIPC & Wingate 1 & Wingate 2 & Wingate 3 & Wingate 4 \\
\hline \multicolumn{5}{|l|}{ Fatigue Index (\%) } \\
\hline Sham Control & $54 \pm 10$ & $55 \pm 12$ & $55 \pm 13$ & $54 \pm 13$ \\
\hline RIPC & $54 \pm 11$ & $55 \pm 11$ & $56 \pm 13$ & $54 \pm 15$ \\
\hline
\end{tabular}

Values are expressed as means $\pm \mathrm{SD}$.

$\mathrm{RIPC}=$ remote ischemic preconditioning

lower body before having subjects complete a running time trial) $[2,3,8,9]$. A recent study reported that IPC applied to the lower limbs delayed fatigue development during repeated handgrip exercise [11]. Our data add novel evidence that remote IPC stimuli can confer significant benefits toanaerobic exercise performance, the task that is highly relevant to athletes competing at any levels. Specifically, we observed an increase in mean power output in the first Wingate test and an increase in peak power output in the fourth Wingate test, demonstrating that bilateral upper extremity RIPC can improve lower-body power output over a series of highly anaerobic exercise challenges. Thus, the present results highlight the potential application and use of RIPC in sports with interval characteristics (e.g., basketball, soccer, ice hockey) that most recreationally-active adults enjoy.

\section{Bilateral IPC: Peak Power (W)}

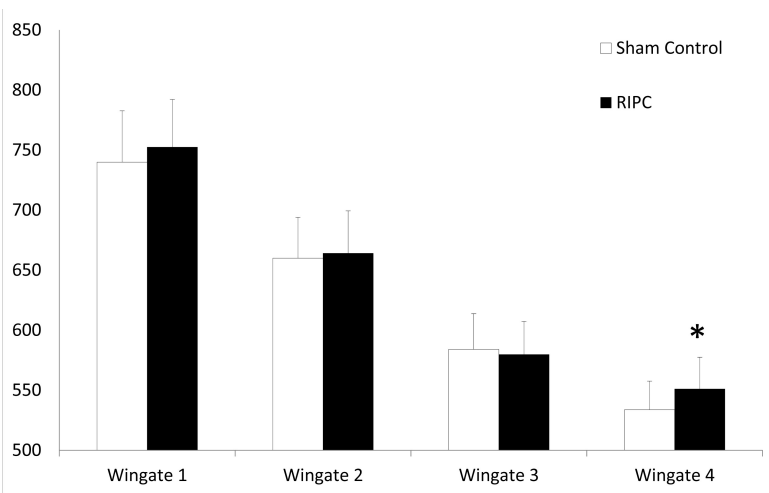

Fig. (2). Peak Wingate anaerobic power in the Sham Control vs Bilateral RIPC groups. Data are presented as means \pm SEM. ${ }^{*} p<0.05$ vs Sham Control.
The previous studies in the field have required the use of a substantially high pressure $(220 \mathrm{mmHg})$ to establish arterial occlusion of the lower body musculature $[2,8,16]$, but even a higher occlusive pressure of $250 \mathrm{mmHg}$ may not fully and completely block arterial flow to the lower extremity [17]. Due to the smaller amount of tissue in the upper extremity, far less pressure is needed for full arterial occlusion. Additionally, in our pilot studies, leg occlusion elicited much greater pain than arm occlusion in most participants. Thus, IPC of the upper extremity employed in the present study provides a more practical, less painful, and widely applicable protocol for a variety of athletes.

Clinical studies have demonstrated a potential benefit of unilateral IPC, as remote preconditioning of one limb prevented subsequent ischemia-reperfusion (IR) induced

\section{Bilateral IPC: Average Power (W)}

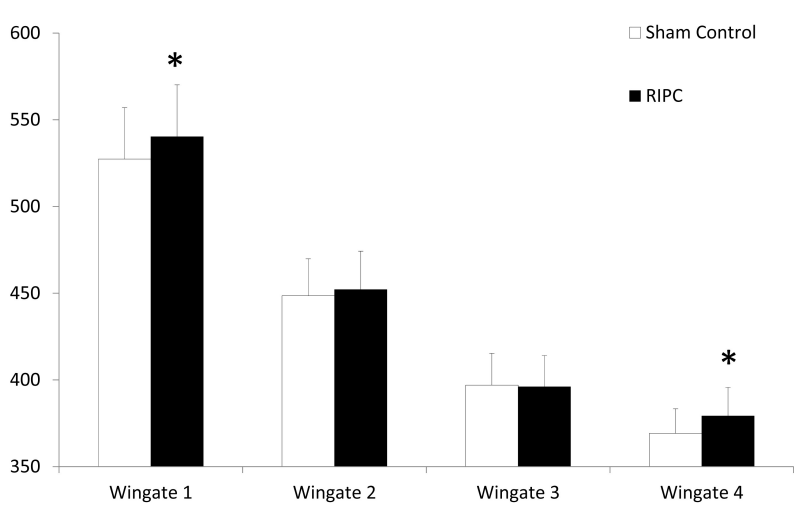

Fig. (3). Mean Wingate anaerobic power in the Sham Control vs Bilateral RIPC groups. Data are presented as means \pm SEM. ${ }^{*} p<0.05$ vs Sham Control. 
Table 3. Composite measures of Wingate anaerobic power outputs and post-exercise blood lactate concentrations.

\begin{tabular}{|l|l|}
\hline \multicolumn{2}{|l|}{ Unilateral RIPC } \\
\hline \hline Total Power Output (W) & $42,435 \pm 8,650$ \\
\hline Sham Control & $41,875 \pm 9,015$ \\
\hline RIPC & $60 \pm 15$ \\
\hline Total Fatigue Index (\%) & $59 \pm 14$ \\
\hline Sham Control & $13.3 \pm 3.2$ \\
\hline RIPC & $13.6 \pm 2.7$ \\
\hline Blood Lactate (mmol/L) & $13.4 \pm 1.4$ \\
\hline Sham Control & $13.0 \pm 2.0$ \\
\hline RIPC & $52,381 \pm 12,932$ \\
\hline Bilateral RIPC & $53,081 \pm 13,429$ \\
\hline \hline Total Power Output (W) & $67 \pm 11$ \\
\hline Sham Control & $66 \pm 12$ \\
\hline RIPC & \\
\hline Total Fatigue Index (\%) & \\
\hline Sham Control & \\
\hline RIPC & \\
\hline Blood Lactate (mmol/L) & \\
\hline Sham Control & \\
\hline RIPC & \\
\hline & \\
\hline
\end{tabular}

Values are expressed as means \pm SD.

$\mathrm{RIPC}=$ remote ischemic preconditioning.

endothelial dysfunction in the contralateral limb [18], and ischemia-reperfusion to one arm produced vasodilatory effects in the brachial artery of the contralateral arm [19]. We reasoned that unilateral IPC would be a more convenient and less painful way to apply RIPC and would produce the effects on anaerobic performance similar to those observed in previous clinical studies [18]. However, unilateral IPC applied remotely had no significant effects on anaerobic performance in the present study. Accordingly, we decided to increase the stimuli by implementing bilateral RIPC with some other changes. In the unilateral IPC trial, we used an occlusion pressure of $10 \mathrm{mmHg}$ for the sham control condition. It is possible that the application of a low pressure cuff may have caused a similar effect of IPC to a greater occlusion pressure as speculated in a previous study [9]. In the bilateral RIPC trial, blood pressure cuff was placed but was not inflated. The results indicated that bilateral RIPC was effective in increasing anaerobic power. The presents study was the first to investigate the dose-response effects of RIPC on exercise performance. These results indicate that a greater amount of IPC would be required to elicit sufficient systemic effects to produce necessary benefit as an ergogenic aid.

The magnitude of improvements in anaerobic exercise performance that we observed in the present study $(2-3 \%)$ was seemingly very small but is in line with the $2-3 \%$ increases reported by previous studies that have addressed the effects of ischemic preconditioning on aerobic performance [2, 8]. Viewed from a biostatistical standpoint, such small increases seem negligible, but athletic competition is often decided by a very small margin of differences. Usain Bolt won the $100 \mathrm{~m}$ sprint in the 2008 Beijing Olympic games in a dominating fashion while setting a new world record, but the difference between the gold and silver medal times in the $100 \mathrm{~m}$ final was only $2 \%$. Thus, from an athletic performance point of view, improvements of $2-3 \%$ observed in the present study may be substantial.

To the best of our knowledge, the present study is the first to investigate the effects of RIPC on "interval exercise" performance. In a previous study demonstrating the benefits of IPC on $100 \mathrm{~m}$ swimming performance, the time trial was conducted 40-45 minutes after the IPC treatment was applied [3]. Additionally, the effects of remote IPC persisted through multiple anaerobic challenges, as a significant increase in anaerobic power was observed in the fourth Wingate test. A longer duration of IPC effects is an important consideration from the standpoint of ergogenic aids as IPC does not have to be performed immediately prior to the sporting events. Currently, it is not known how long the benefits of IPC persist.

What are the physiological mechanisms underlying the effects of RIPC on anaerobic performance? During hypoxic conditions as well as in strenuous exercise, anaerobic energy systems, in particular glycolysis, contribute predominantly to energy production. In the Wingate anaerobic tests, energy from the metabolism of anaerobically-produced lactic acids has been shown to explain $81-83 \%$ of the variances for peak and mean power output [20]. The uncoupling of oxidative phosphorylation by opening mitochondrial ATP-sensitive potassium $\left(\mathrm{K}_{\mathrm{ATP}}\right)$ channels has been suggested to be a physiological mechanism underlying the effect of IPC on reducing oxidative damage and is known to reduce and delay lactate accumulation [21]. However, blood lactate concentrations do not appear to be modulated by IPC in the majority of previous studies [2, 3, 9, 16]. Indeed blood lactate concentration was not different between the RIPC and sham control conditions in the present study.

An alternative explanation is that phosphagen (i.e., ATP and $\mathrm{CP})$ contents in the skeletal muscle may have been elevated by the RIPC stimuli. In initial clinical studies of IPC, better preservation of high energy phosphagens [4] as well as higher muscle contents of ATP and CP [22] occur following preconditioning. The preservation of ATP and reduction in high energy phosphate utilization may contribute to the attenuation of muscle fatigue, and thus the increase in power output, through the modulation of $\mathrm{K}_{\mathrm{ATP}}$ channels. ATP is known to close $K_{\text {ATP }}$ channels [23], and a blockade of $K_{\text {ATP }}$ channels has been shown to attenuate the decline in tetanic forces in fatigued muscle $[24,25]$. Thus, it is likely that the increase and preservation of ATP produced by preconditioning may contribute to an attenuation of the opening of $\mathrm{K}_{\mathrm{ATP}}$ channels to an extent so as to reduce muscle fatigue during a series of Wingate tests. This scenario, however, has not been directly and experimentally tested.

There were a number of limitations inherent in the present study that should be emphasized. As in other previous studies, we could not completely blind our subjects. Although participants remained naïve to the rationale of the experiment, the subjects were undoubtedly aware of pressure differences 
between conditions and could have introduced psychological elements. Additionally, we did not involve a third treatment group, involving ischemic preconditioning of the lower extremity. This type of "local" IPC protocol would have allowed full comparisons of remote and local IPC using identical exercise protocols. Therefore, we cannot fully establish whether one treatment method is preferable over another. Lastly, given a previous finding that the effect of IPC may be attenuated in older adults, the results of our present study cannot be applied to older athletes until similar studies are done in older individuals.

\section{PRACTICAL APPLICATIONS AND CONCLUSION}

Similar to elite athletes, recreational athletes or weekend warriors are always looking for a competitive edge. Ischemic preconditioning has been used to protect myocardial cells against ischemia-reperfusion injury and is recently utilized for improving exercise performance. Because this procedure provides a greater resistance and tolerance to ischemic stress, it appears that most effective venue for the utilization of this technique is anaerobic exercise events. In the present study, a remote ischemic preconditioning protocol applied bilaterally to the upper extremity in healthy, recreationally active individuals increased lower body power output over a series of Wingate anaerobic tests. These results indicate that bilateral RIPC can be an effective means to improve anaerobic power outputs for the purpose of an ergogenic aid. Unilateral RIPC, however, had no effect on any of the performance variables, suggesting that there is a threshold for the amount of target tissue needed to elicit anaerobic performance benefits.

\section{CONFLICT OF INTEREST}

The authors confirm that this article content has no conflict of interest.

\section{ACKNOWLEDGMENTS}

The present study was supported in part by the Ischemic Therapeutic Conditioning, Inc. RAK and HT serve in the scientific advisory boards for the Ischemic Therapeutic Conditioning, Inc. Part of this article has been reproduced from the master thesis of the first author at the University of Texas at Austin.

\section{REFERENCES}

[1] Tokish JM, Kocher MS, Hawkins RJ. Ergogenic aids: a review of basic science, performance, side effects, and status in sports. Am J Sports Med 2004; 32: 1543-53.

[2] de Groot PC, Thijssen DH, Sanchez M, et al. Ischemic preconditioning improves maximal performance in humans. Eur J Appl Physiol 2010; 108: 141-6.

[3] Jean-St-Michel E, Manlhiot C, Li J, et al. Remote preconditioning improves maximal performance in highly trained athletes. Med Sci Sports Exerc 2011; 43: 1280-86.
[4] Murry CE, Jennings RB, Reimer KA. Preconditioning with ischemia: a delay of lethal cell injury in ischemic myocardium. Circulation 1986; 74: 1124-36.

[5] Birnbaum Y, Hale SL, Kloner RA. Ischemic preconditioning at a distance: reduction of myocardial infarct size by partial reduction of blood supply combined with rapid stimulation of the gastrocnemius muscle in the rabbit. Circulation 1997; 96: 1641-6.

[6] Kloner RA. Clinical application of remote ischemic preconditioning. Circulation 2009; 119: 776-8.

[7] Przyklenk K, Bauer B, Ovize M, et al. Regional ischemic 'preconditioning' protects remote virgin myocardium from subsequent sustained coronary occlusion. Circulation 1993; 87: 893-9.

[8] Bailey TG, Jones H, Gregson W, et al. Effect of ischemic preconditioning on lactate accumulation and running performance. Med Sci Sports Exerc 2012; 44: 2084-9.

[9] Crisafulli A, Tangianu F, Tocco F, et al. Ischemic preconditioning of the muscle improves maximal exercise performance but not maximal oxygen uptake in humans. J Appl Physiol 2011; 111: 530-36.

[10] Sabbagh S, Henry Salzman MM, Kloner RA, et al. Remote ischemic preconditioning for coronary artery bypass graft operations. Ann Thorac Surg 2013; 96: 727-36.

[11] Barbosa TC, Machado AC, Braz ID, et al. Remote ischemic preconditioning delays fatigue development during handgrip exercise. Scand J Med Sci Sports 2014; doi: 10.1111/sms.12229.

[12] Tanaka H, Bassett DR Jr, Swensen TC, et al. Aerobic and anaerobic power characteristics of competitive cyclists in the United States Cycling Federation. Int J Sports Med 1993; 14: 334-8.

[13] Souissi N, Driss T, Chamari K, et al. Diurnal variation in Wingate test performances: influence of active warm-up. Chronobiol Int 2010; 27: 640-52.

[14] Vaiksaar S, Jurimae J, Maestu J, et al. No effect of menstrual cycle phase and oral contraceptive use on endurance performance in rowers. J Strength Cond Res 2011; 25: 1571-8.

[15] Bailey TG, Birk GK, Cable NT, et al. Remote ischemic preconditioning prevents reduction in brachial artery flow-mediated dilation after strenuous exercise. Am J Physiol Heart Circ Physiol 2012; 303: H5338.

[16] Clevidence MW, Mowery RE, Kushnick MR. The effects of ischemic preconditioning on aerobic and anaerobic variables associated with submaximal cycling performance. Eur J Appl Physiol 2012; 112: 364954.

[17] Iida H, Kurano M, Takano H, et al. Hemodynamic and neurohormonal responses to the restriction of femoral blood flow by KAATSU in healthy subjects. Eur J Appl Physiol 2007; 100: 275-85.

[18] Kharbanda RK, Mortensen UM, White PA, et al. Transient limb ischemia induces remote ischemic preconditioning in vivo. Circulation 2002; 106: 2881-3.

[19] Enko K, Nakamura K, Yunoki K, et al. Intermittent arm ischemia induces vasodilation of the contralateral upper limb. J Physiol Sci 2011; 61: 507-13.

[20] Beneke R, Pollmann C, Bleif I, et al. How anaerobic is the Wingate anaerobic test for humans? Eur J Appl Physiol 2002; 87: 388-92.

[21] Fryer R, Eells JT, Hsu AK, et al. Ischemic preconditioning in rats: role of mitochondrial K(ATP) channel in preservation of mitochondrial function. Am J Physiol Heart Circ Physiol 2000; 278: 305-12.

[22] Pang CY, Neligan P, Xu H, et al. Role of ATP-sensitive $\mathrm{K}^{+}$channels in ischemic preconditioning of skeletal muscle against infarction. Am J Physiol Heart Circ Physiol 1997; 273: H44-51.

[23] Baukrowitz T, Fakler BK. ATP channels: linker between phospholipid metabolism and excitability. Biochem Pharmacol 2000; 60: 735-40.

[24] Duty S, Allen D. The effects of glibenclamide on tetanic force and intracellular calcium in normal and fatigued mouse skeletal muscle. Exp Physiol 1995; 80: 529-41.

[25] Gramolini A, Renaud J. Blocking ATP-sensitive $\mathrm{K}^{+}$channel during metabolic inhibition impairs muscle contracility. Am J Physiol Cell Physiol 1997; 272: C1936-46. 